\title{
Dwelling in prolonged grief: Resting state functional connectivity during oxytocin and placebo administration
}

\author{
Saren H. Seeley ${ }^{1}$ () | Jessica R. Andrews-Hanna ${ }^{2}$ | John J. B. Allen ${ }^{2}$ () | \\ Mary-Frances O'Connor ${ }^{2}$ (1)
}

${ }^{1}$ Icahn School of Medicine at Mount Sinai, New York City, New York, USA

${ }^{2}$ The University of Arizona, Tucson, Arizona, USA

\section{Correspondence}

Saren H. Seeley, Department of Psychiatry, Icahn School of Medicine at Mount Sinai, 1399 Park Ave., NY 10029, USA.

Email: saren.seeley@mssm.edu

Funding information

Dana Foundation; NIH National Institute of Mental Health, Grant/Award Number: T32MH122394; NIH National Institute on Aging, Grant/Award Number: F31AG062067

\begin{abstract}
Clinical theories of adaptation in bereavement highlight a need for flexible shifting between mental states. However, prolonged motivational salience of the deceased partner may be a complicating factor, particularly when coupled with perseverative thinking about the loss. We investigated how prolonged grief symptoms might relate to resting state functional brain network connectivity in a sample of older adults ( $n=38$ ) who experienced the death of a partner 6-36 months prior, and whether intranasal oxytocin (as a neuropeptide involved in pair-bonding) had differential effects in participants with higher prolonged grief symptoms. Higher scores on the Inventory of Complicated Grief (ICG) were associated with lower anticorrelation (i.e., higher functional connectivity) between the default retrosplenial - cingulo-opercu$\operatorname{lar}_{\mathrm{dACC}}$ network pair. Intranasal oxytocin increased functional connectivity in the

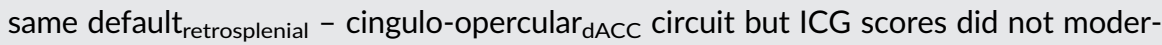
ate effects of oxytocin, contrary to our prediction. Higher ICG scores were associated with longer dwell time in a dynamic functional connectivity state featuring positive correlations among default, frontoparietal, and cingulo-opercular networks, across both placebo and oxytocin sessions. Dwell time was not significantly affected by oxytocin, and higher prolonged grief symptoms were not associated with more variability in dynamic functional connectivity states over the scan. Results offer preliminary evidence that prolonged grief symptoms in older adults are associated with patterns of static and time-varying functional network connectivity and may specifically involve a default network-salience-related circuit that is sensitive to oxytocin.

KEYWORDS

prolonged grief, resting state, functional connectivity, oxytocin, bereavement, dynamic functional connectivity, complicated grief
\end{abstract}

\section{1 | INTRODUCTION}


most people, but some continue to experience intense, impairing grieving without apparent resolution in sight (Prigerson et al., 2021). The condition of severe, chronic grieving (previously: "complicated grief") is currently known as "prolonged grief" or "prolonged grief disorder." Effective coping draws on the capacity to flexibly shift focus between loss- and restoration-related stressors (Stroebe \& Schut, 1999; Stroebe et al., 2010). However, the time scale at which this oscillation should take place (and the measurement thereof), remains to be satisfactorily established (Stroebe et al., 2010). Potentially, we could gain insight into how the bereavement adaptation process goes awry by examining the organization and functioning of large-scale brain networks.

Perseverative, self-referential thoughts interfere with grief adaptation, and contribute to distress (e.g., Eisma et al., 2020; Maccallum \& Bryant, 2013; Robinaugh \& McNally, 2013). One specific complicating process occurs when bereaved people rely on avoidance to manage emotional pain; with the unfortunate outcome that avoidance reinforces monitoring for, and thus salience of, deceased-related cues (Robinaugh et al., 2014; Schneck et al., 2017, 2018).

A second important complicating factor in grief is thought to be prolonged, frustrative proximity-seeking (Kakarala et al., 2020; LeRoy et al., 2019). Intense yearning for reunion is mediated by oxytocin (Hurlemann \& Scheele, 2016; Pohl et al., 2018; Schiele et al., 2018), which regulates attachment formation and maintenance (e.g., Bosch \& Young, 2018) and heightens attention to both aversive and appetitive social stimuli (Bartz et al., 2010, 2011; Eckstein et al., 2014; ShamayTsoory \& Abu-Akel, 2016). Exogenous oxytocin altered resting state functional connectivity among networks that regulate internal versus external focus (e.g., salience, attention, and default networks), and broadly increased cortical-subcortical functional connectivity (Seeley et al., 2018), with a recent study highlighting greater dynamic effective connectivity within salience network regions and from precuneus and posterior cingulate (i.e., default network) to salience network regions in their oxytocin-treated group (Jiang et al., 2021). Oxytocin's ability to elicit this kind of temporary "functional rewiring" likely underlies its effects on self-referential cognition (Liu et al., 2013, 2017).

There is growing evidence for differences in large-scale network function associated with grief severity and prolonged grief, in line with evidence from other affective disorders that similarly feature symptoms like emotional distress and perseverative thinking (Kaiser et al., 2015). Interindividual differences in default network and frontotemporoparietal connectivity are associated with avoidance coping, selective attention to loss cues, and intrusion monitoring in grief (Liu et al., 2015; Schneck et al., 2017, 2018). Earlier cross-sectional fMRI studies demonstrated correlations between yearning and BOLD activation in salience and reward circuit regions, including anterior cingulate and nucleus accumbens (McConnell et al., 2018; O'Connor et al. 2008). In a recent longitudinal study, bereaved adults who displayed greater amygdala functional connectivity with salience and executive control networks experienced worsening grief symptoms over time. Cross-sectionally, higher prolonged grief symptoms were associated with greater amygdala functional connectivity with posterior default network regions (Chen et al., 2020). Further, treatment-seeking bereaved adults who received 8 weeks of mindfulness-based cognitive therapy showed decreased resting state functional connectivity between salience, default, and executive control networks (Huang et al., 2021). Thus, the present study sought to investigate whether oxytocin and self-referential cognition have interactive effects on prolonged grief symptoms, potentially via effects on functional network connectivity during unconstrained thought.

We developed hypotheses informed by Christoff et al. (2016) dynamic framework for spontaneous thought, which proposes a role for flexible network coupling in shaping thoughts' focus and timecourse via both "automatic" (or involuntary) and "deliberate" (or intentional) constraints. Importantly, automatic constraints can be internal (e.g., a strong emotion; self-relevance), not only external stimuli. Prolonged grief perhaps involves more automatic constraints over thought content: the continued salience of mental representations of the deceased and/or their death could underlie the prolonged emotional pain, intense yearning, and/or the intrusive nature of thoughts about the loss that characterize prolonged grief (Robinaugh et al., 2016). Thoughts in prolonged grief might also be less variable over time, given that grief-related rumination likely involves core midline default network regions that have been implicated in other forms of maladaptive self-referential thought (e.g., Fonzo \& Etkin, 2017; Zhou et al., 2020) and other disorders characterized by high levels of perseveration and distress show reduced dFNC variability (e.g., Demirtaş et al., 2016; Kaiser et al., 2016). Operantly defined, higher levels of prolonged grief symptoms might be reflected in greater affective (automatic) constraints over thought content, and less variability, as evidenced by (1) positive correlation between salience network and midline default network time courses (Menon, 2011) and (2) fewer transitions between different dFNC "states" (transient, yet recurrent functional network configurations, and/or longer dwell time in one dFNC state. Given the role of cognitive control in deliberately guiding ones' focus, we also hypothesized that individuals with prolonged grief might show weaker functional connectivity of the frontoparietal network with salience and default networks. While static or time-averaged, functional network connectivity could test the first hypothesis, the latter requires capturing temporal variability that might provide additional insight to the timeinvariant observations via dynamic functional connectivity methods (Kucyi et al., 2018; Lurie et al., 2020).

The current study leveraged existing data from a parent study, in which older adults with varying levels of prolonged grief symptoms participated in two fMRI sessions each as part of a within-subjects crossover design that included oxytocin as a mechanistic probe (ClinicalTrials.gov Identifier: NCT04505904). At one session, they received intranasal oxytocin and at the other, a placebo. Our first aim (data from placebo sessions only) was to identify whether static (FNC) and/or dynamic (dFNC) resting state functional network connectivity was associated with prolonged grief symptoms. We hypothesized that (1A) participants with higher levels of prolonged grief symptoms would show fewer dFNC state transitions over time, and (1B) participants with higher levels of prolonged grief symptoms 
would show higher overall functional connectivity between default and salience networks, lesser functional connectivity with the frontoparietal network, and longer dwell time in a state featuring positive FNC between salience and default networks. Our second aim (data from both sessions) was to investigate intranasal oxytocin's effect on FNC and dFNC in an older, bereaved sample, and to evaluate prolonged grief symptoms as a potential moderator of these effects. We hypothesized that $(2 \mathrm{~A})$ oxytocin (vs. placebo) would increase salience network functional connectivity with the other large-scale networks (default and frontoparietal) hypothesized to be related to prolonged grief symptoms, and (2B) oxytocin will have greater influence on FNC and/or dFNC for participants with higher levels of prolonged grief symptoms.

\section{2 | METHOD}

\section{1 | Participants}

Participants were 40 community-dwelling older adults recruited from the southern Arizona area. All were between 55 and 80 years of age $(M=69.22$ years, $S D=6.49$, and range $=57-79)$ and had experienced the death of a spouse or long-term romantic partner 636 months prior to participation ( $M=15.40$ months and $S D=8.17$ ) Stratified sampling ensured that a full range of ICG scores was represented $(M=23.35, S D=12.47$, and range $=4-51)$. All female participants were postmenopausal.

\subsection{Design and procedures}

All aspects of the study were approved by the University of Arizona's Institutional Review Board Human Subjects Protection Program. Participants who passed a phone screening interview gave written informed consent and were compensated $\$ 200$ for completing the study. Enrolled participants also provided personal photos of their spouse and a living loved one for use in a behavioral task for the parent study.

Participants underwent two scan sessions, a week apart. At each session, they received a double-blinded nasal spray (oxytocin/placebo) and completed a brief measure of their current emotional state. After nasal spray administration followed by a 30-min wait (to allow for oxytocin serum rise-time), structural and functional MRI were performed with total scan time of $\sim 35 \mathrm{~min}$. The resting state sequence was always last, preceded by the structural image acquisition and a behavioral task (Arizmendi et al., n.d.) in which participants viewed photos of their deceased partner, a living loved one, and generic death-related scenes (e.g., hospital room, casket, and gravestone). Resting state began around 68 min after nasal spray administration. The latency did not significantly differ between oxytocin and placebo sessions (see Supporting Information s11). For the resting state scan, participants were instructed to stay awake while a fixation cross was presented on the screen, and "let [their] thoughts come and go as they normally do, but please do not engage in any specific mental activity, such as counting or meditating" to prevent sleep and movement, and encourage participants to allow their mind to wander as it typically would during times of unconstrained thought (Benjamin et al., 2010).

\section{3 | Materials and measures}

\subsection{1 | Measures}

Enrolled participants completed the Beck Depression Inventory-II (BDI-II) (Beck et al., 1996) and the Inventory of Complicated Grief (ICG; Prigerson et al., 1995) from their home computer via Qualtrics before they could attend the first fMRI session. Participants who did not have an email address or preferred not to complete the measures on a computer were mailed paper copies and a prepaid business envelope. They had to return the completed surveys to the lab before they could participate in their first fMRI session. Each participant completed the ICG and BDI-II only once. The ICG is a 19-item measure of prolonged grief symptoms distinct from depression or anxiety which showed high internal consistency in our sample (Cronbach's alpha $=0.93$ ). At the beginning of each scan session (before nasal spray administration), participants completed the state version of the Positive and Negative Affect Schedule (PANAS; Watson et al., 1988), which measures momentary affect along two subscales (positive affect; PANAS-PA, and negative affect; PANAS-NA).

\subsection{2 | Intranasal spray}

At each visit, participants received a double-blinded nasal spray containing either intranasal oxytocin or placebo. A single dose (24 IU; MacDonald et al., 2011) of synthetic oxytocin (Syntocinon, Novartis, Switzerland) or the placebo (Novartis, Switzerland: all nonactive ingredients of Syntocinon) was self-administered via six 4-IU puffs into each nostril, alternating nostrils between each puff. All participants received both oxytocin and placebo sprays. At the second $\mathrm{fMRI}$ session, they received whichever of the nasal sprays was not given at their first session (i.e., if they were assigned to get oxytocin at Session 1, they would get the placebo at Session 2-or vice versa).

\subsection{3 | Randomization}

After a participant was enrolled, a research assistant in the lab (who was not involved in the study in any other capacity) used a random number generator to determine the order in which the new participant would be administered the nasal sprays. Nasal spray vials were labeled "A" and "B" and the research assistant was the only one who knew the vials' true identity until data collection for the parent study was finished. 


\subsection{4 $\quad$ MRI acquisition parameters}

Imaging data were acquired on a Siemens Magnetom Skyra 3 T MRI scanner with 32-channel head coil (Syngo MR E11 software, field strength 2.89) at Banner University Medical Center in Tucson, AZ. A seven-minute structural T1-weighted MPRAGE sequence preceded the functional scans (TR $=2300 \mathrm{~ms}$, TE $=2.3 \mathrm{~ms}, \mathrm{TI}=900 \mathrm{~ms}$, flip angle $=8^{\circ}$, matrix size $=256 \times 256,0.9 \times 0.9 \times 0.9 \mathrm{~mm}$ voxels, 192 slices). Resting state fMRI data were collected during a 6-min, single-echo EPI sequence with 180 contiguous whole-brain functional volumes $\left(\mathrm{TR}=2000 \mathrm{~ms}, \mathrm{TE}=30 \mathrm{~ms}\right.$, flip angle $=90^{\circ}$, matrix $=92 \times 80,2.6 \times 2.6 \times 3.5 \mathrm{~mm}$ voxels, 29 slices).

\section{4 | MRI data preprocessing}

Two of the 40 participants were excluded from further analysis due to excessive movement and/or incomplete brain coverage. Preprocessing used fMRIPrep 1.1.8 (Esteban et al., 2018, 2019; RRID:SCR_ 016216), which is based on Nipype 1.1.3 (Gorgolewski et al., 2018, 2011; RRID:SCR_002502). fMRI data underwent nonaggressive denoising via ICA-AROMA during preprocessing. A detailed description of the preprocessing pipeline and procedures is provided in Supporting Information .

\section{5 | MRI data postprocessing: Group ICA}

\subsection{1 | Group ICA}

We used the Group ICA of fMRI Toolbox (GIFT) 4.0b (Rachakonda et al., 2007) to decompose the imaging data into components representing "functional networks" via group spatial ICA (GICA). GICA back-reconstructs individual subject spatial maps and time courses from the group-level data reduction and ICA estimates and is shown to be more robust than GICA2 or GICA3 for low model order data (Rachakonda et al., 2007). Data reduction incorporated both experimental sessions, with "session" as a repeated (oxytocin or placebo) so that ICs at each session could be directly compared.

ICA was run iteratively 10 times using both random initial values and bootstrapping using ICASSO in GIFT (Himberg \& Hyvärinen, 2003). Spatial maps and time courses for each session and subject were then backreconstructed, or estimated, using GICA (Calhoun et al., 2001), and scaled to Z-scores. A covariance matrix representing static FNC was estimated from Pearson correlation coefficients obtained by averaging the time course correlation between every IC pair (Figure S3). Additional details of data reduction, postprocessing and component labeling approach are included in Supporting Information (p. s14).

Independent components (ICs) at the group level were selected in a two-step process. First, ICs were classified as artifact, mixed/undetermined, or probable resting state network based on their spatial distribution, power spectra, and ICASSO stability estimates (Allen et al., 2011; see also Supporting Information p. s14 for details). Nineteen of the 30 ICs were classified as resting state networks (Table S1; Figure S3). Of the $19 \mathrm{ICs}$, we identified six components corresponding to the three large-scale networks of interest in the current study (default, salience, and frontoparietal; Figure 1): default retrosplenial

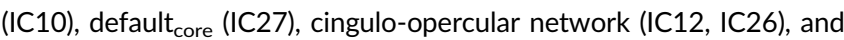
frontoparietal network (IC6, IC17). Note that, we decided to re-label the network for ICs involving anterior insula and dorsal ACC, based on anatomical distribution (i.e., cingulo-opercular network) to avoid assumptions about their function in responding to salient stimuli (as no stimuli were presented during resting state). Second, we examined the individual component maps at each session for the six components. Two more ICs were subsequently excluded. Although the spatial maps for IC12 and IC17 initially appeared to be adequate, a closer examination revealed signal from nonbrain sources: very low fALFF illustrated by a second peak in the high frequency range, which typically indicates noise from other sources (IC12) and the spatial map for the oxytocin session showing eyeball-related movement (IC17).

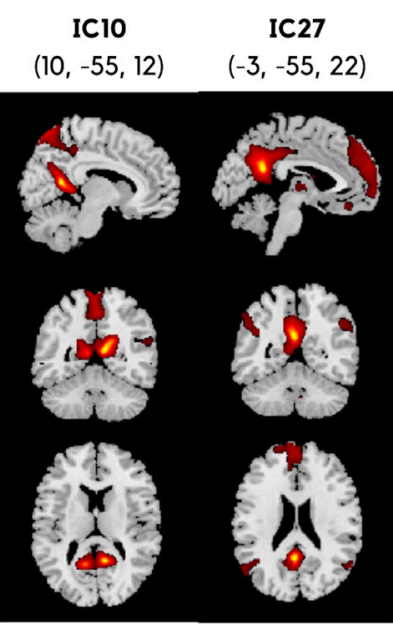

Default network
$\mathrm{ICl} 2$
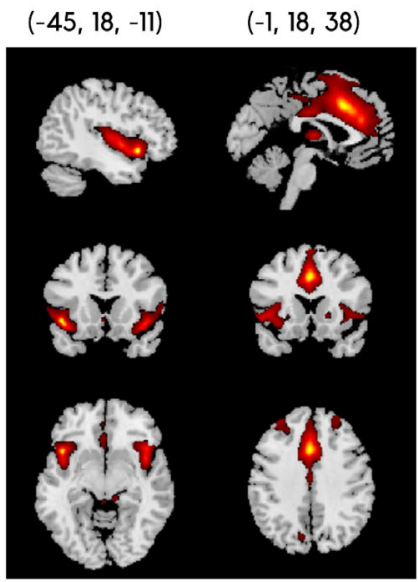

Cingulo-opercular network

IC26

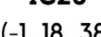

IC6

$(48,-55,58)$

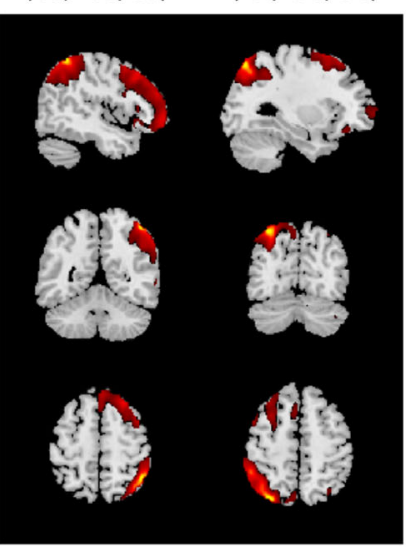

Frontoparietal network
FIGURE 1 Spatial maps of ICs comprising selected resting state networks from the group ICA, thresholded at $Z=2$ and displayed on a standard T1 template image. ICs 10 (default $t_{\text {retrosplenial) }}$ ), 27 (default $_{\text {core }}$ ), 26 (cinguloopercular ${ }_{\mathrm{dACC}}$ ), and 6 (right frontoparietal), were selected for FNC analyses based on their signal quality and relevance to the current study aims 
ICs 10 (default $_{\text {retrosplenial }}$ ), 27 (default core ), 26 (cingulo-opercular ${ }_{\mathrm{dACC}}$ ), and 6 (right frontoparietal), were retained based on their signal quality and relevance to the current study aims.

\section{6 | MRI data postprocessing: Dynamic functional network connectivity (dFNC)}

dFNC "states" represent transient yet recurrent functional network configurations from which additional temporal metrics can be derived. The following postprocessing steps were conducted via the dFNC Toolbox (dFNC v1.0a; Allen et al., 2012) as implemented in GIFT (Rachakonda et al., 2007). A detailed description and validation of the dFNC approach used here is available in Allen et al. (2014). Data from both sessions were analyzed together so that we could compare the same states under oxytocin versus placebo. States were estimated from subject-specific ICA timecourses, using a tapered sliding window of 22 TRs convolved with a Gaussian $\sigma=3$ TRs slid in steps of 1 TR (155 windows per session). The choice of window length was based on the approach described in Allen et al. (2014), in which a tapered 44-second window achieved the best compromise in resolving dynamics versus providing the best estimate of the covariance matrix. Time windows with local maxima in functional connectivity variance ("subject exemplars") were chosen by subsampling windows/pairs for each subject. $k$-means clustering was applied to individual subject matrices representing changes in correlation as a function of time. The clustering analysis yielded four cluster centroids (covariance patterns), or dynamic "states," with the number of clusters chosen based on the elbow criterion for different $k$ values. A representative dFNC matrix for each state was calculated on each subject by averaging $\mathrm{dFNC}$ matrices of that state. Figure 2 shows centroids for the four $\mathrm{dFNC}$ states derived using $k$-means clustering. Participants transitioned between states on average about eight times ( $S D=3.4$ ) over the course of the 6-min resting state scan. State 1 was the most frequently visited state, with 4955 (42\%) centroid occurrences across the sample. State 1 was characterized by weaker covariance among ICs, which tended not to vary much over time except for the cinguloopercular $_{\mathrm{dACC}}$ - frontoparietal $\left.\right|_{\mathrm{R}}$ pair. In State 2 (17\%, or 2025 centroid
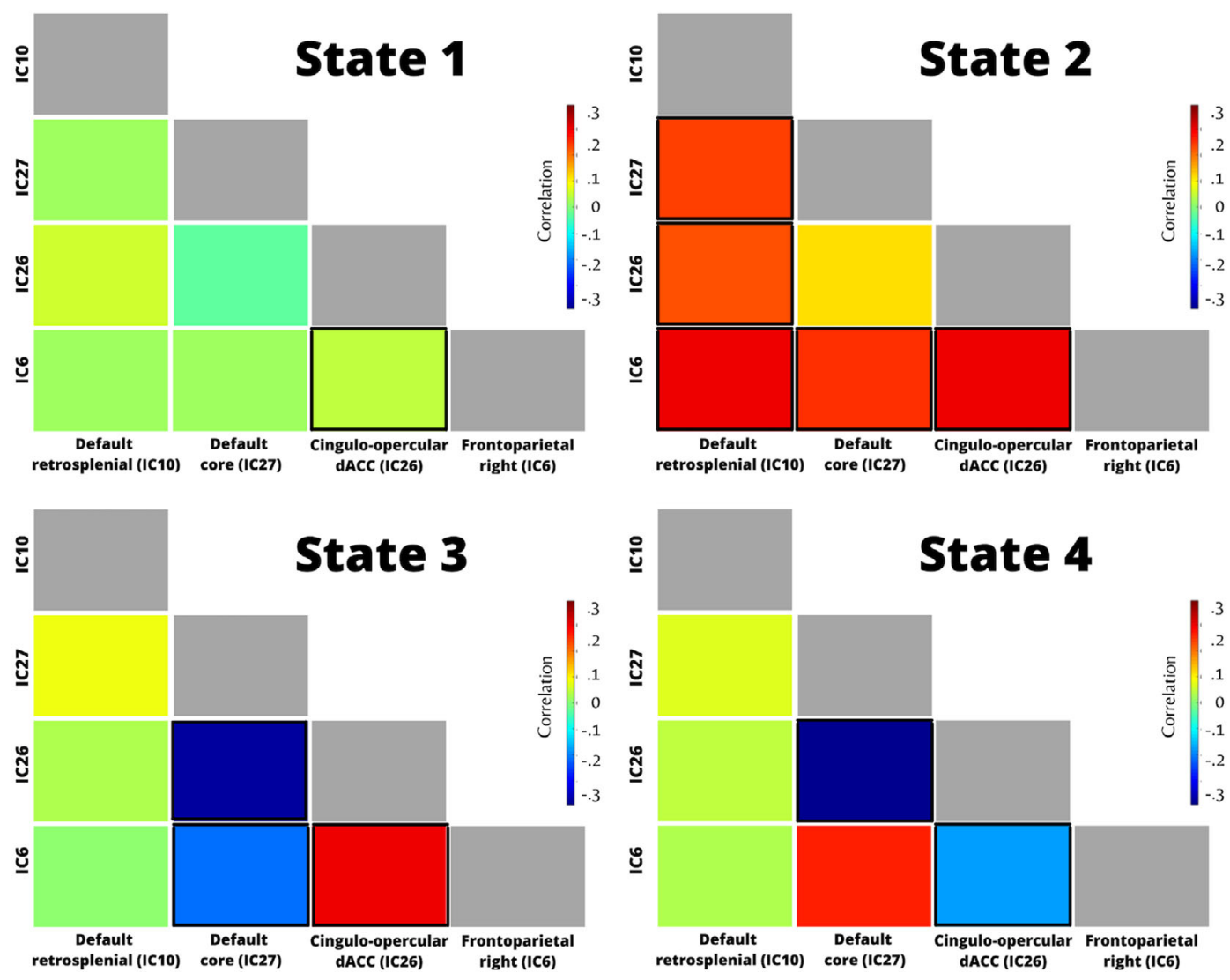

FIGURE 2 K-means clustering applied to windowed covariance matrices (averaged over sessions) yielded four discrete "states," or patterns of functional network configuration. Colors indicate correlation strength and direction. Black outline indicates significantly more variation in the pair's covariance compared to the centroid median (i.e., one-sample $t$-test), pFDR $=.05$. State 1 shows weaker and less variable covariance among ICs. State 2 shows widespread positive correlations among all four network components, with covariance between default core $_{\text {and cingulo- }}$ opercular $_{\mathrm{dACC}}$ being relatively more stable across time. State 3 shows default core $_{\text {anticorrelation with both cingulo-opercular }}$ dAcc $_{\text {and }}$ frontoparietal $_{R}$ components, which were positively correlated with each other. State 4 differed from State 3 in that default core $_{\text {remained }}$

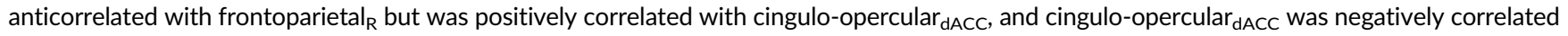
with frontoparietal $I_{R}$ in State 4 
occurrences), participants showed widespread positive timecourse correlation among all four network components, with covariance between default $t_{C o r e}$ and cingulo-opercular $\mathrm{dACC}_{\mathrm{AC}}$ being relatively more stable across time. State 3 ( $22 \%$, or 2557 centroid occurrences) featured default $t_{\text {core }}$ anticorrelation with cingulo-opercular ${ }_{\mathrm{dACC}}$ and frontoparietal $\mathrm{R}_{\mathrm{R}}$ components, which were positively correlated with each other. State 4 (19\%, or 2243 centroid occurrences) differed from State 3 in that default ${ }_{\text {core }}$ remained anticorrelated with frontoparietal ${ }_{R}$ but was positively correlated with cingulo-opercular ${ }_{\mathrm{dACC}}$, and cinguloopercular $_{\mathrm{dACC}}$ was negatively correlated with frontoparietal $\left.\right|_{\mathrm{R}}$.

\subsection{1 $\quad$ dFNC metrics}

Metrics of interest for this study were dwell time, or the average duration that an individual stays in a given state before transitioning to a different state, and total number of transitions ( $n$ transitions), or the number of times an individual moves in and out of different states across the duration of the scan. We calculated these variables for each participant/session based on the results of the $k$-means clustering.

\subsection{Statistical analyses: Approach}

All analyses described in this section were conducted in $\mathrm{R}$ version 1.4.1717 (R Core Team, 2021). Because this was an initial pilot study, we did not correct for multiple comparisons based on the number of statistical tests. However, we sought to minimize the number of comparisons where possible (e.g., through the feature selection approach described below).

\subsection{1 | FNC variables}

We selected a subset of model-related network pairs from the static FNC output. Pairs involving IC17 (frontoparietal $L_{\llcorner}$) were not included as the spatial map for that component showed residual eye movement-related artifact. In addition, our theoretical model based on Christoff et al. (2016) had no specific prediction about the relationship between default $t_{\text {retrosplenial }}$ and frontoparietal networks, so this pair was not included in any analyses. This left eight network pairs: (1) default retrosplenial $_{\text {- default }}$ core, (2) default retrosplenial $_{\text {- cingulo-oper- }}$ cular $_{\text {dACC, (3) default }}$ core - cingulo-opercular ${ }_{\mathrm{dACC}}$, (4) default retrosplenial - cingulo-opercular ${ }_{\text {antInsula, (5) default }}$ core - cingulo-opercular ${ }_{\text {antInsula, }}$ (6) frontoparietal $R_{R}$ - default core, $_{\text {, }}$ (7) frontoparietal ${ }_{R}$ - cingulo-opercu$\operatorname{lar}_{\mathrm{dACC}}$, (8) frontoparietal $\mathrm{R}_{\mathrm{R}}$ - cingulo-opercular ${ }_{\text {antInsula. Additionally, we }}$ had five dFNC variables: dwell time in States 1-4, and $n$ transitions.

\subsection{2 | Covariates}

Age and sex can influence functional connectivity (e.g., Damoiseaux, 2017) and intranasal oxytocin response (e.g., Ebner et al., 2016; Jiang et al., 2021). BDI-II total scores were included as a third covariate, as people with more prolonged grief symptoms tended to endorse more symptoms on the depression measure. Finally, we included pre-scan state positive and negative affect (PANAS-NA, PANAS-PA), mean framewise displacement in each session, and psychoactive medication use as other variables that could be related both to ICG score and FNC.

\subsection{3 | Feature selection}

Prior to testing our hypotheses, we used a data-driven method to select variables for inclusion in the subsequent analyses (described in the Results section). Specifically, we leveraged elastic net regression (Zou \& Hastie, 2005) to identify variables that were robustly related to ICG score, given many candidate FNC variables $(n=13)$ and covariates $(=7)$. In standard multiple regression, OLS parameter estimates are highly variable and thus less reliable in models with too many or correlated variables and smaller $n$, as in our data. Regularized regression approaches such as LASSO (L1 regularization) and ridge (L2 regularization) reduce this variance but at the cost of increasing bias in the coefficient estimates. Elastic net regression is a third form of regularization that seeks to find the optimal tradeoff between bias and variance, which should reduce overfitting and increase out-ofsample predictive accuracy even for smaller data sets. Elastic net regression is useful for data-driven feature selection as it shrinks coefficients to zero for variables that are not robustly related to the outcome variable.

We used the glmnet package in $\mathrm{R}$ (Friedman et al., 2010) to run a 10 -fold cross-validated elastic net with grid search across values of alpha values in .002 increments $(0=$ ridge and LASSO $=1)$ and lambda values to identify parameters of the minimum-MSE model using the placebo-session data. The model with smallest MSE included nonzero coefficients for six of the 20 independent variables: (1) BDIII, (2) state negative affect, (3) sex, $(4,5)$ two static FNC variables (default $_{\text {retrosplenial }}$ - cingulo-opercular ${ }_{\mathrm{dACC}} \mathrm{FNC}$, default $\mathrm{c}_{\text {core }}$ - cinguloopercular $_{\mathrm{dACC}} \mathrm{FNC}$ ), and (6) one dFNC variable (mean dwell time in State 2$), r^{2}=0.70$, indicating that those variables were robustly related to ICG scores. Note that, the regularized regression approach used here does not generate conventional statistics for NHST (e.g., $p$ values); the subsequent analyses provide NHST statistics characterizing the relationship between ICG scores and the "sparse model" variables.

\subsection{4 | Static FNC}

For our first aim (is FNC associated with prolonged grief severity?), we used multiple linear regression predicting ICG score from the two static FNC variables (default retrosplenial - $_{\text {cingulo-opercular }}$ dACC, $_{\text {, }}$ default $_{\text {core }}$ - cingulo-opercular ${ }_{\mathrm{dACC}}$ ) and three covariates (BDI-II, state negative affect, sex) to test whether higher prolonged grief symptoms were associated with FNC between default and cingulo-opercular 
TABLE 1 Participant characteristics

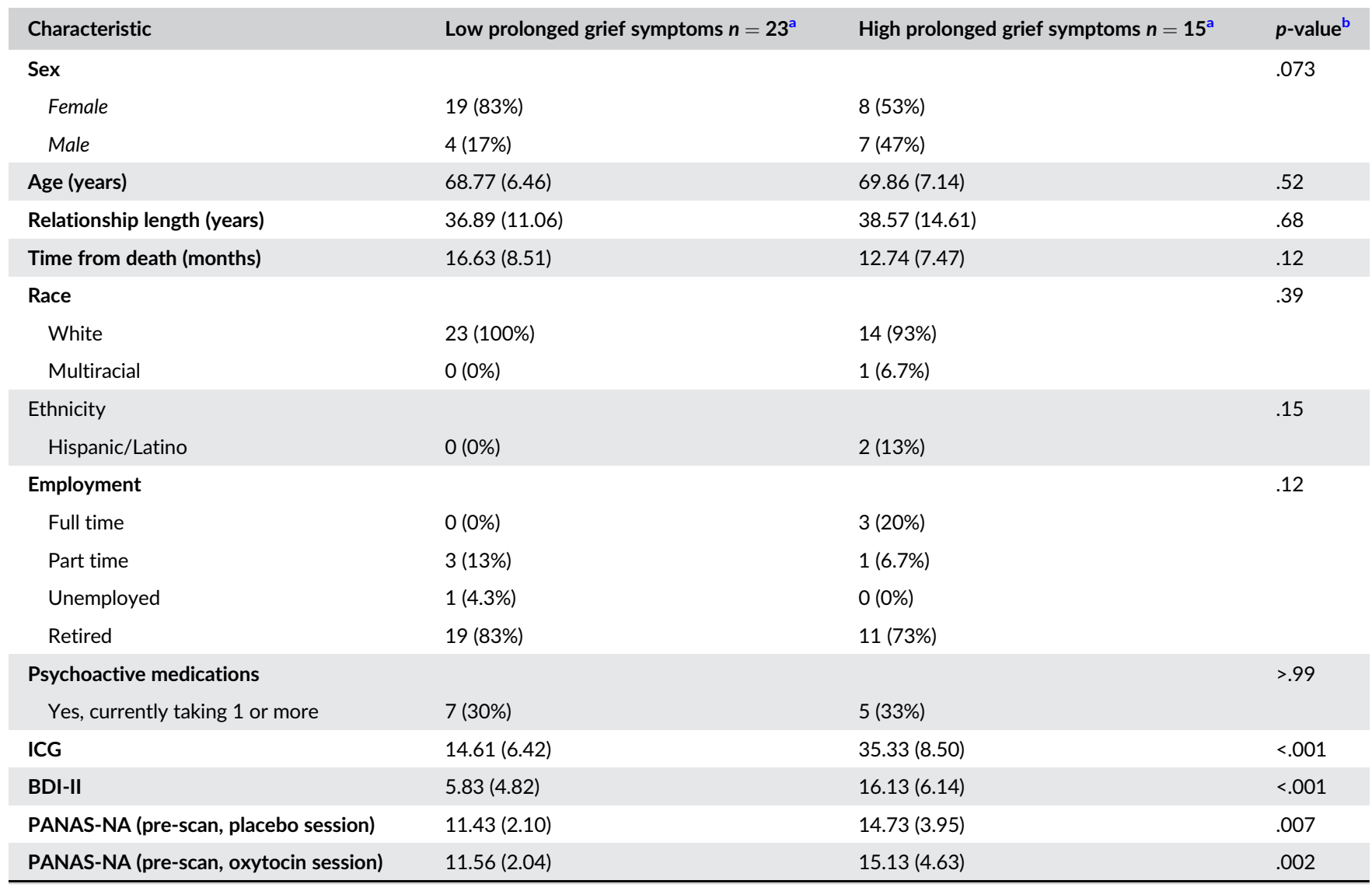

Abbreviations: BDI-II, beck depression linventory-II; ICG, inventory of complicated grief; PANAS-NA, positive and negative affect schedule-state negative affect.

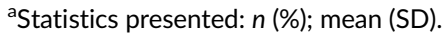

${ }^{b}$ Statistical tests performed: Fisher's exact test; Wilcoxon rank-sum test. In the parent study, enrolled participants were categorized as belonging to either a "low prolonged grief symptoms" or "high prolonged grief symptoms" group, based on a clinical cutoff of ICG $\geq 25$ (Prigerson et al., 1995).

networks. Because the frontoparietal $\left.\right|_{R}$ - cingulo-opercular ${ }_{\mathrm{dACC}} \mathrm{FNC}$ variable was not retained during feature selection, we did not conduct further analyses examining FNC in this pair.

For our second aim (does intranasal oxytocin moderate the relationship between FNC and prolonged grief symptoms?), we used linear mixed effects models with restricted maximum likelihood, which involved session (oxytocin/placebo) and ICG score as fixed effects, random effect of participant, correlation coefficient between a given network pair as outcome, and the same three covariates. We ran two models, examining cingulo-opercular ${ }_{\mathrm{dACC}}$ FNC with default retrosplenial $_{\text {and default }}$ core. For each component pair, Model 1 included only fixed effects of ICG score and Session (placebo/oxytocin) with random effect of participant. Model 2 added BDI-II score, state negative affect, and sex as additional fixed effects.

\subsection{5 | Dynamic FNC}

We used similar multiple regression and linear mixed effects models as described above but with dwell time in State 2 as the outcome for the mixed effects model, rather than static FNC.

\section{$3 \mid$ RESULTS}

\section{1 | Participant characteristics}

The sample ( $n=38$; Table 1 and Figure S1) were primarily female, retired, non-Hispanic White adults, 36 of whom had been in heterosexual partnerships. Most had been in multi-decade relationships and participated more than 1 year after their partner's death. Males were overrepresented in the sample high-prolonged grief symptoms group (i.e., ICG $\geq 25 ; n=15$ ) relative to those with low prolonged grief symptoms (ICG <25; $n=23$ ), but other characteristics (e.g., age, relationship duration, time since death, race, ethnicity, and presence of psychoactive medication use) were similar.

\subsection{Static FNC results}

\subsection{1 | Associations between FNC and prolonged grief symptoms}

Functional connectivity between $\mathrm{DN}_{\text {retrosplenial }}$ and cingulo-operculardAcc components (as an independent variable) was statistically 
significantly associated with ICG scores (dependent variable) in the placebo session data, $b=10.85,95 \% \mathrm{Cl}=[0.67,21.02], \mathrm{SE}=5$, $t=2.17, p=.037$, as were two covariates: male sex, $b=8.27,95 \%$ $\mathrm{Cl}=[2.91,13.62], \mathrm{SE}=2.63, t=3.14, p=.004$, and BDI-II score, $b=1.03,95 \% \mathrm{Cl}=[0.59,1.47], \mathrm{SE}=0.22, t=4.78, p<.001$. The independent variable for $\mathrm{DN}_{\text {core }}$-cingulo-opercular ${ }_{\mathrm{dACC}}$ functional connectivity was not statistically significant in the model, $b=6.61$, $95 \% \mathrm{Cl}=[-0.69,13.92], \mathrm{SE}=3.59, t=1.84, p=.074$, nor was the covariate for state negative affect, $b=0.43,95 \% \mathrm{Cl}=[-0.56,1.41]$, $\mathrm{SE}=0.48, t=0.88, p=.385)$. The overall model with covariates explained approximately two-thirds of the variance in ICG scores (F $(5,32)=17.10$, adjusted $R^{2}=0.69, p=<.001$.

\subsubsection{Effects of intranasal oxytocin and prolonged grief symptoms on FNC}

When we tested joint effects of intranasal oxytocin and prolonged grief symptoms (independent variables) on FNC (dependent variable), defaul $_{\text {retrosplenial }}$ - cingulo-opercular ${ }_{\mathrm{dACC}}$ was the only component pair with a significant difference between the oxytocin and placebo sessions. Specifically, their FNC was higher in the oxytocin session (Table S2; Figure S4). The effect of oxytocin remained after including BDI-II score, sex, and state negative affect as covariates in the model. Contrary to our hypothesis, there was no ICG score $x$ Session interaction. Oxytocin had no apparent effect, alone or interaction, on FNC between default core $_{\text {- cingulo-opercular }}$ dAcc components (Table S3).

\section{3 | Dynamic FNC results}

\subsection{1 | Associations between $\mathrm{dFNC}$ and prolonged grief symptoms}

Dwell time in State 2 (independent variable) was positively associated with ICG scores (dependent variable) in the placebo session data, $b=0.2,95 \% \mathrm{Cl}=[0,0.41], \mathrm{SE}=0.1, t=2.05, p=.049$ - along with the covariates male sex, $b=6.56,95 \% \mathrm{Cl}=[0.64,12.48]$, SE $=2.91$, $t=2.25, p=.031$, and BDI-II score, $b=0.94,95 \% \mathrm{Cl}=[0.5,1.38]$ $\mathrm{SE}=0.22, t=4.33, p<.001$. State negative affect was not statistically significant when included as a covariate, $b=0.62,95 \%$ $\mathrm{Cl}=[-0.4,1.64], \mathrm{SE}=0.5, t=1.24, p=.225$. The overall model with covariates explained about two-thirds of the variance, $F$ $(4,33)=18.12$, adjusted $R^{2}=0.65$, and $p<.001$.

\subsection{2 | Effects of intranasal oxytocin and prolonged grief symptoms on dFNC}

When we tested joint effects of intranasal oxytocin and prolonged grief symptoms (independent variables) on dFNC State 2 (dependent variable), we identified that intranasal oxytocin did not significantly impact dwell time in State 2 (Figure 3 and Table S4). The association
Mean Dwell Time in dFNC State by Group and Session

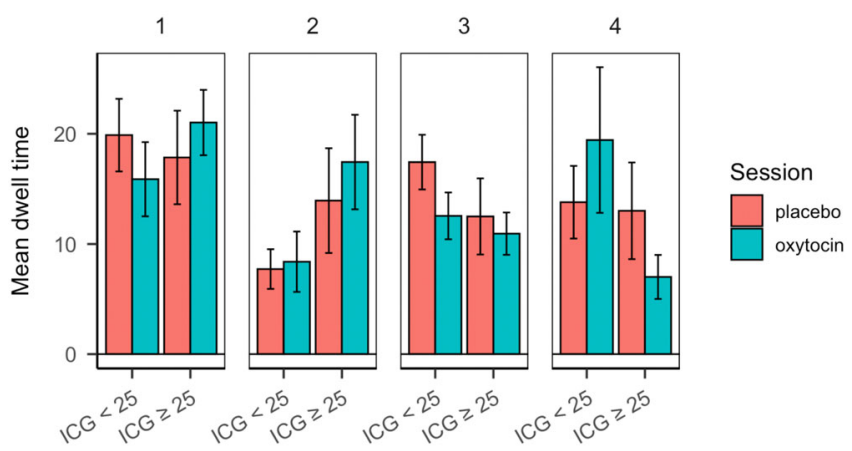

FIGURE 3 Mean dwell time in each dynamic functional connectivity state, by group and session. Participants with high prolonged grief symptoms (ICG score $\geq 25$ ) group spent more time in State 2 than those with low prolonged grief symptoms (ICG score $<25$ ), across both oxytocin + placebo sessions

between higher ICG scores and longer State 2 dwell time remained statistically significant in the combined oxytocin+placebo session data, but contrary to our hypothesis, there was no ICG score $\times$ Session interaction.

\section{4 | DISCUSSION}

\section{1 | Summary and implications}

The present study aimed to identify effects of prolonged grief symptoms and intranasal oxytocin on large-scale brain network connectivity during resting state, using a data-driven approach for network detection. We identified a network pair (default retrosplenial $_{\text {and cingulo- }}$ opercular $_{\mathrm{dACC}}$ ) in which higher resting state functional connectivity in the placebo session was associated with fewer prolonged grief symptoms. Intranasal oxytocin increased functional connectivity in the same similar circuit (default retrosplenial and cingulo-opercular ${ }_{\mathrm{dACC}}$ ), though the effect of oxytocin was not moderated by prolonged grief symptoms (contrary to our hypothesis).

The default $t_{\text {retrosplenial }}$ and cingulo-opercular ${ }_{\mathrm{dACC}}$ components both involve structures in which OXTR mRNA is highly expressed (Quintana et al., 2019). Similar to our findings, an earlier gICA study found that intranasal oxytocin increased resting state functional connectivity between networks identified as "salience network AcC" and "ventral posterior default network" in healthy adults (Xin et al., 2018), with the unthresholded spatial map for their ventral posterior default network closely resembling the component labeled as "default retrosplenial" in our study. Although our theoretical model focused on the salience network, we ultimately labeled the putatively salience-related components in terms of their anatomy (i.e., as part of the cinguloopercular network) rather than presumed function. The labels "salience network" and "cingulo-opercular network" are often used interchangeably, and both appear to be largely anchored in the dorsal ACC and anterior insula. However, they may in fact represent distinct, 
though closely located networks that serve different functions. The salience network connectivity is involved in bottom-up stimulus capture, whereas the cingulo-opercular network is involved in attentional switching and maintenance to facilitate cognitive control via frontoparietal network connectivity (Power et al., 2011). The distinction between the two networks is an important one for prolonged grief, as Power et al. (2011) description of cingulo-opercular network function seems more relevant than bottom-up stimulus processing in exploring how bereaved people regulate the focus of their thoughts. The spatial maps labeled in the present study as "default" and "cingulo-opercular" also resemble meta-analytic correlates of transdiagnostic perseverative thought, with $\mathrm{AACC}$ and precuneus activation differentiating clinical and healthy control groups (Makovac et al. 2020), though we note that our findings implicate the medial temporal default network subsystem rather than the $\mathrm{mPFC} /$ precuneus/posterior cingulate core default network. Together, these findings may further support (1) prolonged grief as a syndrome in which prolonged salience of the deceased/their death and unconstrained thought (perhaps involving autobiographical memories) are mutually reinforcing, and (2) a possible role for oxytocin in brain regions linked to prolonged grief symptoms (Arizmendi et al., n.d.; Kakarala et al., 2020). However, we note that our prediction that prolonged grief symptoms would be associated with less variability in dFNC as measured by lower $n$ transitions was not supported by these data. Also contrary to our hypothesis, intranasal oxytocin did not have a differential effect on participants with higher prolonged grief symptoms during resting state. The relationship requires further probing given that oxytocin did have differential effects on behavior in the same sample (Arizmendi et al., n.d.): oxytocin slowed overall response times in high- (but not low-) prolonged grief symptom groups.

There was no apparent effect of intranasal oxytocin on dynamic functional connectivity, matching recent findings from younger healthy samples in which oxytocin did not modulate dwell time or number of transitions (Jiang et al., 2021). However, consistent with our hypothesis that people with higher prolonged grief symptoms would experience greater automatic (i.e., emotional) constraints over their thoughts during resting state, we found that participants with higher levels of prolonged grief symptoms displayed longer dwell time in a dynamic state characterized by both intra- and inter-default network coupling (State 2). Longer dwell time in State 2 might reflect the tendency for people with prolonged grief to get "stuck" in griefrelated thoughts-particularly after confronting reminders of the deceased, which they did in the task preceding resting state scanning in our study. For example, emotional content was reflected in a dFNC pattern featuring extensive network interconnectivity during unconstrained thought (Wang et al., 2018), and negative emotional content in the preceding task led to greater default network coactivation (Gaviria et al., 2021). Reduced anticorrelation between salience and default networks may reflect off-task intrusions (Kucyi et al., 2018). Higher default network connectivity has also been linked to more vivid memory (Turnbull et al., 2019), and autobiographical memory retrieval is associated with increased correlation between default network and distributed nondefault network regions (Warren et al., 2018), which fits with the $\mathrm{DN}_{\text {retrosplenial }}$ and cingulo-operculardACC FNC seen in our study. Deceased-related mental representations interfere with ongoing attention (Freed et al., 2009; Schneck et al., 2018), though may be adaptive if processing does not rise to the level of conscious awareness and the thoughts are not experienced as intrusive (Schneck et al., 2018). In contrast, prolonged grief involves aspects of both automatic (intrusions) and deliberate constraints on thought (cognitive avoidance via rumination or suppression). Although we did not find any association between prolonged grief symptoms and static FNC in the right frontoparietal network component, its internetwork connectivity in the dFNC State 2 might reflect efforts in bereaved people to monitor and guide mental activity. Indeed, this network component resembles a cognitive controlrelated subnetwork involved in regulating internal thoughts and emotions (vs. regulating external attention) through interactions with the default network (Dixon et al. 2018).

\section{2 | Limitations}

Our results should be interpreted in the context of several limitations. First, many of our findings are modest and exploratory (despite clear theory-driven hypotheses) and we did not correct for the total number of statistical tests. Data presented here are cross-sectional, so we cannot identify whether differences in static and dynamic functional connectivity represents a trait vulnerability or reflect the instantiation of processes involved in poorer adaptation-a consequence or correlate, rather than a determinant of risk for prolonged grief. In terms of design limitations, the sample of White older adults was not representative of the population-level demographics of United States widows (Granek and Peleg-Sagy, 2017), the parent study did not include ongoing thought assessment during resting state, which would strengthen the interpretation of the function of identified networks, and the placement of resting state after a behavioral task that included photos of the deceased could have influenced the resting state activity (though whether that influence would be desirable or undesirable is debatable).

There are also several important methodological caveats to consider, given outstanding questions and controversies in the field around methodological, statistical, and biological considerations in studying intranasal oxytocin effects, and/or time-varying functional connectivity as it relates to the brain as a dynamic system (Lurie et al., 2020). Although $24 \mathrm{IU}$ is one of the more common supraphysiologic oxytocin doses used for human research, there is mixed evidence for which dose elicits the strongest effects (e.g., $8 \mathrm{IU}$ vs. $24 \mathrm{IU}$ ) and how the uptake of intranasal oxytocin could be affected by individual differences in nasal cavity physiology and thus efficacy of administration (Quintana et al., 2021). There is also mixed evidence for how long the acute effects of neural and behavioral effects of intranasal oxytocin last. One study of resting state cerebral blood flow changes in response to intranasal oxytocin versus placebo identified a pharmacodynamic peak at the 39-51 min period; however, the effect of oxytocin was sustained across the full 78-min duration of the study 
(Paloyelis et al., 2016). Participants in this study began the resting state scan at $68 \mathrm{~min}$ on average, and it is possible that the null effects on $\mathrm{dFNC}$ in the present study were a consequence of the later timing or using an improper dosage.

Dynamic (time-varying) functional connectivity measures may suffer from undesirably low test-retest reliability (Choe et al. 2017; Zhang et al., 2016), which is particularly relevant in the context of our repeated-measures study design. However, cortical networks, particular default and frontoparietal, do seem to be more reliable than subcortical networks (Noble et al., 2019). Both structural and functional brain connectivity measures have been critiqued for sensitivity to motion and physiological artifacts so to mitigate potential for spurious correlations, we used ICA-AROMA (Pruim, Mennes, Rooij, et al. 2015; Pruim, Mennes, Buitelaar, et al. 2015), which performs better than standard motion parameter regression with less data loss than volume censoring (Parkes et al., 2018). Dynamic functional connectivity estimation may be influenced by parameters such as sliding window length and cut-off frequency (Leonardi \& Van De Ville, 2015). This issue is complicated by the fact that we do not know the ground truth for issues like "How long does a thought last?" (and thus, what is the most accurate window length?) Longer window lengths may obscure briefer changes, and vice versa. For our data, potential sliding window lengths lay between 100 and $6.67 \mathrm{~s}$ (based on the lowest and highest frequencies of interest at $0.01-0.15 \mathrm{~Hz}$ ), but window lengths of $30 \mathrm{~s}$ and above may be optimal for the tapered sliding window approach used here (Xie et al., 2019), though our 44-s window could miss any very slow dynamics (i.e., between 0.01 and $023 \mathrm{~Hz}$ ). We chose the 22-TR window (44 s) following Allen et al. (2014) observation that $44 \mathrm{~s}$ achieved a good tradeoff between the ability to resolve dynamics and covariance matrix estimation quality. Finally, varying the value of $k$ in the $k$-means clustering approach might have yielded different results. Here, we selected $k$ using a mathematical criterion (given our lack of principled empirical predictions for how many states "should" be expected), which Allen et al. (2014) showed to provide results consistent with those across a wide range of $k$. However, testing whether results replicated across a range of parameter values would have inspired greater confidence in our findings.

\section{5 | CONCLUSION}

This study illustrates differences in static and dynamic resting state functional connectivity measures in bereaved older adults representing a wide range of prolonged grief symptoms. Higher prolonged grief symptoms were associated with increased inter-network functional connectivity, particularly between retrosplenial default and cinguloopercular network regions. The same retrosplenial default cinguloopercular was sensitive to intranasal oxytocin manipulation. Though modest, our data support the idea that both brain network connectivity and the neuropeptide oxytocin may play roles in prolonged grief. The observed effect of oxytocin on default network - cinguloopercular network static functional connectivity in our bereaved sample is intriguing given theoretical conceptualizations of reward in prolonged grief disorder (Kakarala et al., 2020). However, it is important to emphasize that intranasal oxytocin was used here as a mechanistic probe: this study is not equipped to study the therapeutic value of intranasal oxytocin, nor does it establish a rationale for using intranasal oxytocin to treat prolonged grief symptoms. Future studies should seek to establish whether the functional connectivity differences observed here replicate in younger bereaved adults, whether they are present pre-morbidly or only following loss, and if they reflect specific mental processes (e.g., differences in thought form or content) in people experiencing prolonged grief. Given that grief adaptation is a process that unfolds over time (across both long- and short-term scales), dynamic functional connectivity remains an intriguing tool but would be most informative in the context of careful study design that can capture ongoing changes in subjective experience.

\section{AUTHOR CONTRIBUTIONS}

Mary-Frances O'Connor conceptualized, designed the parent study, obtained grant funding, and data collection. Saren H. Seeley developed the current study's hypotheses and approach, led data analyses, data collection, obtained grant funding, and drafted the original manuscript. John J. B. Allen contributed substantially to study resources, hypothesis refinement, data analysis, and interpretation of results. Jessica Andrews-Hanna contributed substantially to study resources, hypothesis refinement, data analysis, and interpretation of results. All authors contributed to and approved the final study manuscript.

\section{ACKNOWLEDGMENTS}

The authors wish to thank Brian Arizmendi and Scott Squire for their integral contributions to the parent study, and Elena Plante for her feedback on earlier versions of this project.

\section{CONFLICT OF INTEREST}

The author declares that there is no conflict of interest that could be perceived as prejudicing the impartiality of the research reported.

\section{DATA AVAILABILITY STATEMENT}

The data are not publicly available due to privacy or ethical restrictions.

\section{ETHICS STATEMENT}

All aspects of the study were approved by the University of Arizona's Institutional Review Board Human Subjects Protection Program. Participants provided written informed consent prior to participation and were debriefed after they completed their study.

\section{FUNDING}

This work was supported by the National Institutes of Health (NIH), the National Institute on Aging (F31AG062067), the National Institute on Mental Health (T32MH122394), and by a Neuroscience Research Grant from the DANA Foundation. This content is solely the responsibility of the authors and does not necessarily represent the official views of the NIH, NIA, NIMH, or the DANA Foundation. 


\section{ORCID}

Saren H. Seeley (D) https://orcid.org/0000-0002-9493-8512

John J. B. Allen (D) https://orcid.org/0000-0002-3417-6720

Mary-Frances O'Connor (DD https://orcid.org/0000-0001-5961-6350

\section{REFERENCES}

Allen, E. A., Damaraju, E., Plis, S. M., Erhardt, E. B., Eichele, T., \& Calhoun, V. D. (2014). Tracking whole-brain connectivity dynamics in the resting state. Cerebral Cortex, 24(3), 663-676.

Allen, E. A., Erhardt, E. B., Damaraju, E., Gruner, W., Segall, J. M., Silva, R. F., Havlicek, M., Rachakonda, S., Fries, J., Kalyanam, R., Michael, A. M., Caprihan, A., Turner, J. A., Eichele, T., Adelsheim, S., Bryan, A. D., Bustillo, J., Clark, V. P., Ewing, S. W. F., ... Calhoun, V. D. (2011). A baseline for the multivariate comparison of resting-state networks. Frontiers in Systems Neuroscience, 5, 2. https://doi.org/10. 3389/fnsys.2011.00002

Allen, E. A., Erhardt, E. B., Wei, Y., Eichele, T., \& Calhoun, V. D. (2012). Capturing inter-subject variability with group independent component analysis of fMRI data: A simulation study. Neuroimage, 59, 41414159. https://doi.org/10.1016/j.neuroimage.2011.10.010

Arizmendi, B., Seeley, S., Allen, J., Killgore, W., Andrews-Hanna, J., \& O'Connor, M. (under review). Feeling a pull to be close: The effect of oxytocin on approach and avoidance in prolonged grief.

Bartz, J. A., Zaki, J., Bolger, N., \& Ochsner, K. N. (2011). Social effects of oxytocin in humans: Context and person matter. Trends in Cognitive Sciences, 15(7), 301-309. https://doi.org/10.1016/j.tics.2011.05.002

Bartz, J. A., Zaki, J., Ochsner, K. N., Bolger, N., Kolevzon, A., Ludwig, N., \& Lydon, J. E. (2010). Effects of oxytocin on recollections of maternal care and closeness. Proceedings of the National Academy of Sciences of the United States of America, 107(50), 21371-21375. https://doi.org/ 10.1073/pnas.1012669107

Beck, A., Steer, R., \& Brown, G. (1996). Beck depression inventory-II (BDI-II). Springer.

Benjamin, C., Lieberman, D. A., Chang, M., Ofen, N., Whitfield-Gabrieli, S., Gabrieli, J. D., \& Gaab, N. (2010). The influence of rest period instructions on the default mode network. Frontiers in Human Neuroscience, 4, 218. https://doi.org/10.3389/fnhum.2010.00218

Bosch, O. J., \& Young, L. J. (2018). Oxytocin and social relationships: From attachment to bond disruption. Current Topics in Behavioral Neurosciences, 35, 97-117. https://doi.org/10.1007/7854_2017_10

Calhoun, V. D., Adali, T., Pearlson, G. D., \& Pekar, J. J. (2001). A method for making group inferences from functional MRI data using independent component analysis. Human Brain Mapping, 14(3), 140-151. https://doi.org/10.1002/hbm.1048

Chen, G., Ward, B. D., Claesges, S. A., Li, S.-J., \& Goveas, J. S. (2020). Amygdala Functional Connectivity Features in Grief: A Pilot Longitudinal Study. The American Journal of Geriatric Psychiatry, 28(10), 10891101. https://doi.org/10.1016/j.jagp.2020.02.014

Christoff, K., Irving, Z. C., Fox, K. C. R., Spreng, R. N., \& AndrewsHanna, J. R. (2016). Mind-wandering as spontaneous thought: A dynamic framework. Nature Reviews Neuroscience, 17(11), 718-731. https://doi.org/10.1038/nrn.2016.113

Damoiseaux, J. S. (2017). Effects of aging on functional and structural brain connectivity. Neurolmage, 160, 32-40. https://doi.org/10.1016/ j.neuroimage.2017.01.077

Demirtaş, M., Tornador, C., Falcón, C., López-Solà, M., HernándezRibas, R., Pujol, J., Menchón, J. M., Ritter, P., Cardoner, N., SorianoMas, C., \& Deco, G. (2016). Dynamic functional connectivity reveals altered variability in functional connectivity among patients with major depressive disorder. Human Brain Mapping, 37(8), 2918-2930. https:// doi.org/10.1002/hbm.23215

Dixon, M. L., De La Vega, A., Mills, C., Andrews-Hanna, J., Spreng, R. N., Cole, M. W., \& Christoff, K. (2018). Heterogeneity within the frontoparietal control network and its relationship to the default and dorsal attention networks. Proceedings of the National Academy of Sciences, 115(7). https://doi.org/10.1073/pnas.1715766115

Ebner, N. C., Chen, H., Porges, E., Lin, T., Fischer, H., Feifel, D., \& Cohen, R. A. (2016). Oxytocin's effect on resting-state functional connectivity varies by age and sex. Psychoneuroendocrinology, 69, 50-59. https://doi.org/10.1016/j.psyneuen.2016.03.013

Eckstein, M., Scheele, D., Weber, K., Stoffel-Wagner, B., Maier, W., \& Hurlemann, R. (2014). Oxytocin facilitates the sensation of social stress. Human Brain Mapping., 35, 4741-4750. https://doi.org/10. 1002/hbm.22508

Eisma, M. C., Lang, T. A. d., \& Boelen, P. A. (2020). How thinking hurts: Rumination, worry, and avoidance processes in adjustment to bereavement. Clinical Psychology and Psychotherapy, 27, 548-558. https://doi.org/10.1002/cpp.2440

Esteban, O., Blair, R., Markiewicz, C. J., Berleant, S. L., Moodie, C., Ma, F., Isik, A. I., Erramuzpe, A., Kent, M., James, D., \& Gorgolewski, K. J. (2018). poldracklab/fmriprep: 1.1 .3 (1.1.3). Zenodo. https://doi.org/ 10.5281/zenodo.852659

Esteban, O., Markiewicz, C. J., Blair, R. W., Moodie, C. A., Isik, A. I., Erramuzpe, A., Kent, J. D., Goncalves, M., DuPre, E., Snyder, M., Oya, H., Ghosh, S. S., Wright, J., Durnez, J., Poldrack, R. A., \& Gorgolewski, K. J. (2019). fMRIPrep: A robust preprocessing pipeline for functional MRI. Nature Methods, 16(1), 111-116. https://doi.org/ 10.1038/s41592-018-0235-4

Fonzo, G. A., \& Etkin, A. (2017). Affective neuroimaging in generalized anxiety disorder: An integrated review. Dialogues in Clinical Neuroscience, 19(2), 169-179.

Freed, P. J., Yanagihara, T. K., Hirsch, J., \& Mann, J. J. (2009). Neural mechanisms of grief regulation. Biological Psychiatry, 66(1), 33-40. https:// doi.org/10.1016/j.biopsych.2009.01.019

Friedman, J., Hastie, T., \& Tibshirani, R. (2010). Regularization paths for generalized linear models via coordinate descent. Journal of Statistical Software, 33(1), 1-22.

Gaviria, J., Rey, G., Bolton, T., Delgado, J., Van De Ville, D., \& Vuilleumier, P. (2021). Brain functional connectivity dynamics at rest in the aftermath of affective and cognitive challenges. Human Brain Mapping, 42(4), 1054-1069.

Gorgolewski, K., Burns, C. D., Madison, C., Clark, D., Halchenko, Y. O., Waskom, M. L., \& Ghosh, S. (2011). Nipype: A flexible, lightweight and extensible neuroimaging data processing framework in python. Frontiers in Neuroinformatics, 5, 13. https://doi.org/10.3389/fninf.2011. 00013

Gorgolewski, K. J., Esteban, O., Markiewicz, C. J., Ziegler, E., Ellis, D. G., Notter, M. P., Jarecka, D., Johnson, H., Burns, C., Manhães-Savio, A., Hamalainen, C., Yvernault, B., Salo, T., Jordan, K., Goncalves, M., Waskom, M., Clark, D., Wong, J., Loney, F., ... Ghosh, S. (2018). nipy. nipype: 1.1 .3 (1.1.3). Zenodo.. https://doi.org/10.5281/zenodo. 596855

Granek, L., \& Peleg-Sagy, T. (2017). The use of pathological grief outcomes in bereavement studies on African Americans. Transcultural Psychiatry, 54(3), 384-399. https://doi.org/10.1177/1363461517708121

Himberg, J., \& Hyvärinen, A. (2003). ICASSO: Software for investigating the reliability of ICA estimates by clustering and visualization. 2003 IEEE XIII Workshop on Neural Networks for Signal Processing (IEEE Cat. No.03TH8718). https://doi.org/10.1109/NNSP.2003.1318025

Huang, F., Hsu, A., Chao, Y., Shang, C. M., Tsai, J., \& Wu, C. W. (2021). Mindfulness-based cognitive therapy on bereavement grief: Alterations of resting-state network connectivity associate with changes of anxiety and mindfulness. Human Brain Mapping, 42(2), 510-520. https://doi.org/10.1002/hbm.25240

Hurlemann, R., \& Scheele, D. (2016). Dissecting the role of oxytocin in the formation and loss of social relationships. Biological Psychiatry, 79(3), 185-193. https://doi.org/10.1016/j.biopsych.2015.05.013 
Jiang, X., Ma, X., Geng, Y., Zhao, Z., Zhou, F., Zhao, W., Yao, S., Yang, S., Zhao, Z., Becker, B., \& Kendrick, K. M. (2021). Intrinsic, dynamic and effective connectivity among large-scale brain networks modulated by oxytocin. Neuroimage, 227, 117668.

Kaiser, R. H., Andrews-Hanna, J. R., Wager, T. D., \& Pizzagalli, D. A. (2015). Large-scale network dysfunction in major depressive disorder: A metaanalysis of resting-state functional connectivity. JAMA Psychiatry, 72(6), 603-611. https://doi.org/10.1001/jamapsychiatry.2015.0071

Kaiser, R. H., Whitfield-Gabrieli, S., Dillon, D. G., Goer, F., Beltzer, M., Minkel, J., Smoski, M., Ditcher, G., \& Pizzagalli, D. A. (2016). Dynamic resting-state functional connectivity in major depression. Neuropsychopharmacology, 41(7), 1822-1830. https://doi.org/10.1038/npp. 2015.352

Kakarala, S., Roberts, K., Rogers, M., Coats, T., Falzarano, F., Gang, J., Chilov, M., Avery, J., Maciejewski, P. K., Lichtenthal, W. G., \& Prigerson, H. (2020). The neurobiological reward system in prolonged grief disorder (PGD): A systematic review. Psychiatry Research: Neuroimaging, 303, 111135. https://doi.org/10.1016/j.pscychresns.2020. 111135

Kucyi, A., Tambini, A., Sadaghiani, S., Keilholz, S., \& Cohen, J. R. (2018). Spontaneous cognitive processes and the behavioral validation of time-varying brain connectivity. Network Neuroscience, 2(4), 397-417. https://doi.org/10.1162/NETN_a_00037

Leonardi, N., \& Van De Ville, D. (2015). On spurious and real fluctuations of dynamic functional connectivity during rest. Neurolmage, 104, 430436. https://doi.org/10.1016/j.neuroimage.2014.09.007

LeRoy, A. S., Knee, C. R., Derrick, J. L., \& Fagundes, C. P. (2019). Implications for reward processing in differential responses to loss: Impacts on attachment hierarchy reorganization. Personality and Social Psychology Review, 23(4), 391-405. https://doi.org/10.1177/1088868319853895

Liu, W., Liu, H., Wei, D., Sun, J., Yang, J., Meng, J., Wang, L., \& Qiu, J. (2015). Abnormal degree centrality of functional hubs associated with negative coping in older Chinese adults who lost their only child. Biological Psychology, 112, 46-55. https://doi.org/10.1016/j.biopsycho. 2015.09.005

Liu, Y., Sheng, F., Woodcock, K. A., \& Han, S. (2013). Oxytocin effects on neural correlates of self-referential processing. Biological Psychology, 94, 380-387. https://doi.org/10.1016/j.biopsycho.2013.08.003

Liu, Y., Wu, B., Wang, X., Li, W., Zhang, T., Wu, X., \& Han, S. (2017). Oxytocin effects on self-referential processing: Behavioral and neuroimaging evidence. Social Cognitive and Affective Neuroscience, 12, 1845-1858. https://doi.org/10.1093/scan/nsx116

Lurie, D. J., Kessler, D., Bassett, D. S., Betzel, R. F., Breakspear, M., Kheilholz, S., Kucyi, A., Liégeois, R., Lindquist, M. A., Mclntosh, A. R., Poldrack, R. A., Shine, J. M., Thompson, W. H., Bielczyk, N. Z., Douw, L., Kraft, D., Miller, R. L., Muthuraman, M., Pasquini, L., ... Calhoun, V. D. (2020). Questions and controversies in the study of time-varying functional connectivity in resting fMRI. Network Neuroscience, 4(1), 30-69. https://doi.org/10.1162/netn_a_00116

Maccallum, F., \& Bryant, R. A. (2013). A cognitive attachment model of prolonged grief: Integrating attachments, memory, and identity. Clinical Psychology Review, 33(6), 713-727. https://doi.org/10.1016/j.cpr. 2013.05.001

MacDonald, E., Dadds, M. R., Brennan, J. L., Williams, K., Levy, F., \& Cauchi, A. J. (2011). A review of safety, side-effects and subjective reactions to intranasal oxytocin in human research. Psychoneuroendocrinology, 36(8), 1114-1126. https://doi.org/10.1016/j.psyneuen. 2011.02.015

Makovac, E., Fagioli, S., Rae, C. L., Critchley, H. D., \& Ottaviani, C. (2020). Can't get it off my brain: Meta-analysis of neuroimaging studies on perseverative cognition. Psychiatry Research: Neuroimaging, 295 111020. https://doi.org/10.1016/j.pscychresns.2019.111020

McConnell, M. H., Killgore, W. D., \& O'Connor, M. F. (2018). Yearning predicts subgenual anterior cingulate activity in bereaved individuals. Heliyon, 4(10), e00852. https://doi.org/10.1016/j. heliyon.2018.e00852

Menon, V. (2011). Large-scale brain networks and psychopathology: A unifying triple network model. Trends in Cognitive Sciences, 15(10), 483506. https://doi.org/10.1016/J.TICS.2011.08.003

Noble, S., Scheinost, D., \& Constable, R. T. (2019). A decade of test-retest reliability of functional connectivity: A systematic review and metaanalysis. Neurolmage, 203, 116157. https://doi.org/10.1016/j. neuroimage.2019.116157

O'Connor, M.-F., Wellisch, D. K., Stanton, A. L., Eisenberger, N. I., Irwin, M. R., \& Lieberman, M. D. (2008). Craving love? Enduring grief activates brain's reward center. Neurolmage, 42(2), 969-972. https:// doi.org/10.1016/j.neuroimage.2008.04.256

O'Connor, M. F., \& Seeley, S. H. (2021). Grieving as a form of learning: Insights from neuroscience applied to grief and loss. Current Opinion in Psychology, 43, 317-322. https://doi.org/10.1016/j.copsyc.2021. 08.019

Paloyelis, Y., Doyle, O. M., Zelaya, F. O., Maltezos, S., Williams, S. C., Fotopoulou, A., \& Howard, M. A. (2016). A Spatiotemporal Profile of In Vivo Cerebral Blood Flow Changes Following Intranasal Oxytocin in Humans. Biological Psychiatry, 79(8), 693-705. https://doi.org/10. 1016/j.biopsych.2014.10.005

Parkes, L., Fulcher, B., Yücel, M., \& Fornito, A. (2018). An evaluation of the efficacy, reliability, and sensitivity of motion correction strategies for resting-state functional MRI. Neurolmage, 171, 415-436. https://doi. org/10.1016/j.neuroimage.2017.12.073

Pohl, T. T., Young, L. J., \& Bosch, O. J. (2018). Lost connections: Oxytocin and the neural, physiological, and behavioral consequences of disrupted relationships. International Journal of Psychophysiology, 136, 54-63. https://doi.org/10.1016/j.ijpsycho.2017.12.011

Power, J. D., Cohen, A. L., Nelson, S. M., Wig, G. S., Barnes, K. A., Church, J. A., Vogel, A. C., Laumann, T. O., Miezin, F. M., Schlaggar, B. L., \& Petersen, S. E. (2011). Functional Network Organization of the Human Brain. Neuron, 72(4), 665-678. https://doi.org/ 10.1016/j.neuron.2011.09.006

Prigerson, H. G., Maciejewski, P. K., Reynolds, C. F., Bierhals, A. J., Newsom, J. T., Fasiczka, A., Frank, E., Doman, J., \& Miller, M. (1995). Inventory of complicated grief: A scale to measure maladaptive symptoms of loss. Psychiatry Research, 59(1-2), 65-79. https://doi.org/10. 1016/0165-1781(95)02757-2

Prigerson, H. G., Kakarala, S., Gang, J., \& Maciejewski, P. K. (2021). History and status of prolonged grief disorder as a psychiatric diagnosis. Annual Review of Clinical Psychology, 17(1), 109-126. https://doi.org/ 10.1146/annurev-clinpsy-081219-093600

Pruim, R. H. R., Mennes, M., Buitelaar, J. K., \& Beckmann, C. F. (2015). Evaluation of ICA-AROMA and alternative strategies for motion artifact removal in resting state fMRI. Neurolmage, 112, 278-287. https:// doi.org/10.1016/j.neuroimage.2015.02.063

Pruim, R. H. R., Mennes, M., van Rooij, D., Llera, A., Buitelaar, J. K., \& Beckmann, C. F. (2015). ICA-AROMA: A robust ICA-based strategy for removing motion artifacts from fMRI data. Neurolmage, 112, 267-277. https://doi.org/10.1016/j.neuroimage.2015.02.064

Quintana, D. S., Rokicki, J., van der Meer, D., Alnæs, D., Kaufmann, T., Córdova-Palomera, A., Dieset, I., Andreassen, O. A., \& Westlye, L. T. (2019). Oxytocin pathway gene networks in the human brain. Nature Communications, 10(1). https://doi.org/10.1038/s41467-01908503-8

Quintana, D. S., Lischke, A., Grace, S., Scheele, D., Ma, Y., \& Becker, B. (2021). Advances in the field of intranasal oxytocin research: lessons learned and future directions for clinical research. Molecular Psychiatry, 26(1), 80-91. https://doi.org/10.1038/s41380-02000864-7

Rachakonda, S., Egolf, E., Correa, N., Calhoun, V., \& Neuropsychiatry, O. (2007). Group ICA of fMRI toolbox (GIFT) manual. 
R Core Team (2021). R: A language and environment for statistical computing. R Foundation for Statistical Computing, Vienna, Austria. https:// www.R-project.org/

Robinaugh, D. J., LeBlanc, N. J., Vuletich, H. A., \& McNally, R. J. (2014). Network analysis of persistent complex bereavement disorder in conjugally bereaved adults. Journal of Abnormal Psychology, 123(3), 510522. https://doi.org/10.1037/abn0000002

Robinaugh, D. J., Mauro, C., Bui, E., Stone, L., Shah, R., Wang, Y., Skritskaya, N. A., Reynolds, C. F., Zisook, S., O'Connor, M.-F., Shear, K., \& Simon, N. M. (2016). Yearning and its measurement in complicated grief. Journal of Loss and Trauma, 21(5), 410-420. https:// doi.org/10.1080/15325024.2015.1110447

Robinaugh, D. J., \& McNally, R. J. (2013). Remembering the past and envisioning the future in bereaved adults with and without complicated grief. Clinical Psychological Science, 1, 290-300. https://doi.org/10. 1177/2167702613476027

Schiele, M. A., Costa, B., Abelli, M., Martini, C., Baldwin, D. S., Domschke, K., \& Pini, S. (2018). Oxytocin receptor gene variation, behavioural inhibition, and adult separation anxiety: Role in complicated grief. The World Journal of Biological Psychiatry, 19(6), 471-479. https://doi.org/10.1080/15622975.2018.1430374

Schneck, N., Haufe, S., Tu, T., Bonanno, G. A., Ochsner, K. N., Sajda, P., \& Mann, J. J. (2017). Tracking deceased-related thinking with neural pattern decoding of a cortical-basal ganglia circuit. Biological Psychiatry: Cognitive Neuroscience and Neuroimaging, 2(5), 421-429. https://doi. org/10.1016/j.bpsc.2017.02.004

Schneck, N., Tu, T., Michel, C. A., Bonanno, G. A., Sajda, P., \& Mann, J. J. (2018). Attentional bias to reminders of the deceased as compared with a living attachment in grieving. Biological Psychiatry: Cognitive Neuroscience and Neuroimaging, 3(2), 107-115. https://doi.org/10. 1016/j.bpsc.2017.08.003

Seeley, S. H., Chou, Y., \& O'Connor, M.-F. (2018). Intranasal oxytocin and OXTR genotype effects on resting state functional connectivity: A systematic review. Neuroscience \& Biobehavioral Reviews, 95, 17-32. https://doi.org/10.1016/j.neubiorev.2018.09.011

Shamay-Tsoory, S. G., \& Abu-Akel, A. (2016). The social salience hypothesis of oxytocin. Biological Psychiatry, 79(3), 194-202. https://doi.org/ 10.1016/j.biopsych.2015.07.020

Stroebe, M., \& Schut, H. (1999). The dual process model of coping with bereavement: Rationale and description. Death Studies, 23(3), 197224. https://doi.org/10.1080/074811899201046

Stroebe, M., Schut, H., \& Boerner, K. (2010). Continuing bonds in adaptation to bereavement: Toward theoretical integration. Clinical Psychology Review, 30(2), 259-268. https://doi.org/10.1016/j.cpr.2009.11.007

Turnbull, A., Wang, H.-T., Schooler, J. W., Jefferies, E., Margulies, D. S., \& Smallwood, J. (2019). The ebb and flow of attention: Between-subject variation in intrinsic connectivity and cognition associated with the dynamics of ongoing experience. Neurolmage, 185, 286-299. https:// doi.org/10.1016/j.neuroimage.2018.09.069
Wang, H. T., Bzdok, D., Margulies, D., Craddock, C., Milham, M., Jefferies, E., \& Smallwood, J. (2018). Patterns of thought: Population variation in the associations between large-scale network organisation and self-reported experiences at rest. Neuroimage, 176, 518-527.

Warren, K. N., Hermiller, M. S., Nilakantan, A. S., O'Neil, J. Palumbo, R. T., \& Voss, J. L. (2018). Increased fMRI activity correlations in autobiographical memory versus resting states. Human Brain Mapping, 39(11), 4312-4321.

Watson, D., Clark, L. A., \& Tellegen, A. (1988). Development and validation of brief measures of positive and negative affect: The PANAS scales. Journal of Personality and Social Psychology, 54(6), 1063-1070. https:// doi.org/10.1037/0022-3514.54.6.1063

Xie, H., Zheng, C. Y., Handwerker, D. A., Bandettini, P. A., Calhoun, V. D., Mitra, S., \& Gonzalez-Castillo, J. (2019). Efficacy of different dynamic functional connectivity methods to capture cognitively relevant information. Neuroimage, 188, 502-514. https://doi.org/10.1016/j. neuroimage.2018.12.037

Xin, F., Zhou, F., Zhou, X., Ma, X., Geng, Y., Zhao, W., Yao, S., Dong, D., Biswal, B. B., Kendrick, K. M., \& Becker, B. (2018). Oxytocin modulates the intrinsic dynamics between attention-related large-scale networks. Cerebral Cortex, 31, 1848-1860. https://doi.org/10.1093/cercor/ bhy295

Zhang, J., Cheng, W., Liu, Z., Zhang, K., Lei, X., Yao, Y., Becker, B., Liu, Y., Kendrick, K. M., Lu, G., \& Feng, J. (2016). Neural, electrophysiological and anatomical basis of brain-network variability and its characteristic changes in mental disorders. Brain, 139, 2307-2321. https://doi.org/ 10.1093/brain/aww143

Zhou, H. X., Chen, X., Shen, Y. Q., Li, L., Chen, N. X., Zhu, Z. C., Castellanos, F. X., \& Yan, C. G. (2020). Rumination and the default mode network: Meta-analysis of brain imaging studies and implications for depression. Neuroimage, 206, 116287.

Zou, H., \& Hastie, T. (2005). Regularization and variable selection via the elastic-net. Journal of the Royal Statistical Society, 67(2), 301-320. https://doi.org/10.1111/j.1467-9868.2005.00503.x

\section{SUPPORTING INFORMATION}

Additional supporting information can be found online in the Supporting Information section at the end of this article.

How to cite this article: Seeley, S. H., Andrews-Hanna, J. R., Allen, J. J. B., \& O'Connor, M.-F. (2022). Dwelling in prolonged grief: Resting state functional connectivity during oxytocin and placebo administration. Human Brain Mapping, 1-13. https://doi.org/10.1002/hbm.26071 